\title{
Quantum numerical control for particles at matter surface
}

\author{
Quan-Fang Wang* \\ The Chinese University of Hong Kong \\ E-mail: quanfangwang9@gmail.com
}

In this work, time-depended Schrödinger equation described particles at matter (crystal, catalysis, metal) surface could be considered as propose of numerical control of quantum system. Accessing existing physical experimental results on the motion of particles (molecules, atoms) at surface, based on variational method of quantum control theory in Hilbert space, using density function theory (DFT), time-depended Schrödinger equation to proceed the investigation of computational approach. To do quantum calculation at surface, physically, first needs a concept as control goal: such as breaking a chemical bond as target; reducing energy of high intensity shaped laser pulse. Particles at surface is a kind of constrain control for spatial variable. Optimal control is to find and characterize the quantum optima for minimizing or maximizing the cost functional. Control methods contain selecting chemical reagent, designing chemical reaction, making control scope for a quantized system: time varying Schrödinger equation.

Precisely, for general quadratic cost function, in two or three dimensional cases, a semidiscrete (time continuous, spatial discrete) algorithm consisting of finite element method and conjugate gradient method, would be utilized for solving a numerical solution of state system, and obtaining quantum optimal control from a initial guess of control input. It is

\footnotetext{
*To whom correspondence should be addressed
} 
quite curious: what is the difference of control particles occurred at surface than control free particles? whether one can develop a suit of theory or methodology for quantum surface control? It is certainly expected to connect theoretical control, to numerical or computational control, and to experimental control as carrying out quantum system control of particles on the surface. It is desired that quantum control theory (QCT) for quantum dot at surface would be evidenced in visualization method, and attained confidential verification in the guidance of real-time computer-aided experiments in the viewpoint of chemistry and physics.

\section{History of study on particles at surface}

In the studying of particles at the matter surface, initial consideration is reported on the 2009 ACS meeting for quantum control of quantum dot at surface. ${ }^{1}$ That is a work for theoretical and computational control to Klein-Gordon-Schrödinger (KGS) equation for 1 and 2 dimensions cases. At the year 2014 ACS meetings, ${ }^{2,3}$ nano-particles control had been treated using the density function theory (DFT) for the motion of electrons surrounding the particles at surface. Theoretic result is obtained for multi-particles cases. ${ }^{4,6-11,13}$ Lasting the survey on DFT, ${ }^{12}$ this work is manipulating one particle at matter surface in the aspect of numerical approach. The interesting thing is to evidence that control theory is worked for DFT, computational control can be performed for a time-depended Schrödinger equation at real physics gauges. ${ }^{5}$ The significant difference of KGS system and DFT: one is aim at wave function of a particle, and another is aim at electron density of a particle. As perspective research, numerical control in high dimensions for nano-particles at matter surface could be a considerable direction in the future.

\section{General density function theory}

For a particle at surface, introduce density functional theory as preparation. Let $\Omega$ be an open bounded set of $\mathbf{R}^{3}$ at spherical polar coordinate $\mathbf{r} \in(0, \infty)$, vector $\mathbf{r}$ as in Figure 1 , and 
$Q=(0, T) \times \Omega$ for $T>0,(t, \mathbf{r}) \in Q$. The ground state energy density of electron is denoted

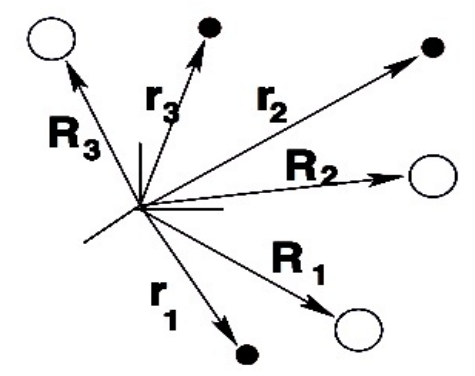

Figure 1: Spherical polar coordinate, $\mathbf{r}=\left(r_{1}, r_{2}, r_{3}\right), \mathbf{R}=\left(R_{1}, R_{2}, R_{3}\right)$.

as $\eta(t, r), \mathbf{r}$ is the displacement of electron from centre of a particle, $\vec{E}(t, \overrightarrow{\mathbf{r}})$ is electronic field. Then time-dependent density functional theory has the form of Schödinger equation

$$
\mathbf{i} \frac{\partial}{\partial t} \eta(t, \mathbf{r})=\left[-\frac{1}{2} \nabla^{2}+\int \frac{\eta\left(t, \mathbf{r}^{\prime}\right)}{\left|\mathbf{r}-\mathbf{r}^{\prime}\right|} d \mathbf{r}^{\prime}+\frac{\delta E_{x c}(\eta(t, \mathbf{r}))}{\delta(t, \mathbf{r}))}-\vec{E}_{0}(t) \cdot \overrightarrow{\mathbf{r}}\right] \eta(t, \mathbf{r})
$$

In here, $\eta(t, \mathbf{r})$ is wave function of the particle, represent the probability function at time $t$. $E_{x c}(\eta(t, \mathbf{r}))$ is exchange correction function. For unified scattered field $\vec{E}_{s}(t, \overrightarrow{\mathbf{r}})$, the Hamiltonian of particle in the presence of incident field $\vec{E}_{0}(t)$ at time-depended density functional theory (TDDFT) lead the form of

$$
\hat{H}(t)=-\frac{1}{2} \nabla^{2}+\int \frac{\eta\left(t, \mathbf{r}^{\prime}\right)}{\left|\mathbf{r}-\mathbf{r}^{\prime}\right|} d \mathbf{r}^{\prime}+\frac{\delta E_{x c}(\eta(t, \mathbf{r}))}{\delta(t, \mathbf{r}))}-\vec{E}_{0}(t) \cdot \overrightarrow{\mathbf{r}}-\vec{E}_{s}(t, \mathbf{r}) \cdot \overrightarrow{\mathbf{r}}
$$

\section{Time depended Schrödinger equation}

For $N$ particles at surface, $\mathbf{n}\left(t, \mathbf{r}^{\prime}\right)=\sum_{i=1}^{N}\left|\eta^{i}(t, \mathbf{r})\right|^{2}$, time-depended Schrödinger equation is expressed as

$$
\mathbf{i} \frac{\partial}{\partial t} \eta^{i}(t, \mathbf{r})=-\frac{1}{2} \nabla^{2} \eta^{i}(t, \mathbf{r})+[e(t, \mathbf{r})+V(t, \mathbf{r})-g(t) \mathbf{r}] \eta^{i}(t, \mathbf{r}), \quad 1 \leq i \leq N
$$


In here, $e(t, \mathbf{r})=\int \frac{\mathbf{n}\left(t, \mathbf{r}^{\prime}\right)}{\left|\mathbf{r}-\mathbf{r}^{\prime}\right|} d \mathbf{r}^{\prime}$ is electron-electron interaction, $V(t, \mathbf{r})=\frac{\delta E_{x c}(\mathbf{n}(t, \mathbf{r}))}{\delta(t, \mathbf{r})}$ is the contribution of potential energy functional $E_{x c}$. For polar vector $\overrightarrow{\mathbf{r}}=\left(r_{1}, r_{2}, r_{3}\right)^{t}$ and $\vec{E}_{0}(t)=(g(t), g(t), g(t))^{t}, \vec{E}_{0}(t) \cdot \overrightarrow{\mathbf{r}}=g(t) \mathbf{r}$ is external electronic field. For different density functional model, $E_{x c}$ term at $V(t, \mathbf{r})$ is expressed as detail of Thomas-Fermi (TF) model, Thomas-Fermi-Dirac (TFD) model, Thomas-Fermi-Dirac-Weizsäcker (TFDW) model.

$$
\begin{aligned}
& V(t, \mathbf{r})=\frac{\delta E_{x c}(\mathbf{n}(t, \mathbf{r}))}{\delta(t, \mathbf{r})} \\
& =\frac{5}{3} c_{k} \int \mathbf{n}(\mathbf{r})^{2 / 3} d \mathbf{r}+v(\mathbf{r}) \\
& =\frac{5}{3} c_{k} \int \mathbf{n}(\mathbf{r})^{2 / 3} d \mathbf{r}+v(\mathbf{r})-\frac{4}{3} c_{e} \int \mathbf{n}(\mathbf{r})^{\frac{1}{3}} d \mathbf{r} \quad \text { TFD model } \\
& =\frac{5}{3} c_{k} \int \mathbf{n}(\mathbf{r})^{2 / 3} d \mathbf{r}+v(\mathbf{r})-\frac{4}{3} c_{e} \int \mathbf{n}(\mathbf{r})^{\frac{1}{3}} d \mathbf{r} \\
& +\frac{1}{8}\left(\frac{h^{2}}{m}\right) \int\left[\frac{\left|N \mathbf{n}(\mathbf{r})^{2}\right|}{\mathbf{n}(\mathbf{r})}-2 \frac{|N \mathbf{n}(\mathbf{r})|}{\mathbf{n}(\mathbf{r})}\right] d \mathbf{r}, \quad \text { TFDW model }
\end{aligned}
$$

$c_{k}=\frac{3}{10}\left(\frac{h}{m}\right)\left(3 \pi^{2}\right)^{2 / 3}$ and $c_{e}=e^{2} \frac{3}{4}\left(\frac{3}{\pi}\right)^{1 / 3}$. For several nucleus, columb potential $v(\mathbf{r})=$ $\sum_{\sigma} \frac{C_{\sigma}}{\left|\mathbf{r}-R_{\sigma}\right|}, C_{\sigma}$ is charge, $\mathbf{r}$ is coordinate of electron at nucleus $R_{\sigma}$. At TFW model, last term of TFDW theory is in the form of gradient expression as $\frac{\hbar}{m} \int(\nabla \mathbf{n})^{1 / 2}(\mathbf{r})^{2} d \mathbf{r} . \quad h, \hbar$ is (reduced) Planck constant, $m$ is electron mass.

\section{Mathematical setting}

To do mathematical setting for time-depended Schrödinger equation (1) in the framework of variational method. For the particle at matter or metal surface, consider complex space for wave function $\eta^{i}(t, \mathbf{r})$ on variable $t$ and $\mathbf{r}$, take real Hilbert space for variational theory. In system (1), $g(t)$ is external force of electron, as control input of particle $P^{i}$. Control function $g(t)$ at incident electric field $\vec{E}_{0}(t)$ is only depended on time $t$, and independent of spatial variable vector $\mathbf{r}$.

To usual spaces $L^{2}(\Omega)$ and $H_{0}^{1}(\Omega)$, define the complex solution space $\mathbb{L}^{2}(\Omega)$ and $\mathbb{H}_{0}^{1}(\Omega)$. 
For complex-valued function $\eta^{i}(t, \mathbf{r})$, denote two complex Hilbert spaces $\mathbb{H}=\mathbb{L}^{2}(\Omega)$ and $\mathbb{V}=\mathbb{H}_{0}^{1}(\Omega)$. For $\eta^{i}=\eta^{i 1}+\mathbf{i} \eta^{i 2} \in \mathbb{L}^{2}(\Omega), \eta^{i 1}, \eta^{i 2} \in L^{2}(\Omega)$, define norm of space $\mathbb{L}^{2}(\Omega)$ as

$$
\left\|\eta^{i}\right\|_{\mathbb{L}^{2}(\Omega)}=\left(\left\|\eta^{i 1}\right\|_{L^{2}(\Omega)}^{2}+\left\|\eta^{i 2}\right\|_{L^{2}(\Omega)}^{2}\right)^{\frac{1}{2}} .
$$

If $\eta^{i}=\eta^{i 1}+\mathbf{i} \eta^{i 2} \in \mathbb{L}^{2}(\Omega), n^{i}=n^{i 1}+\mathbf{i} n^{i 2} \in \mathbb{L}^{2}(\Omega)$, then the inner product of $\mathbb{L}^{2}(\Omega)$ can be defined by

$$
\left(\eta^{i}, n^{i}\right)_{\mathbb{L}^{2}(\Omega)}=\left(\left(\eta^{i 1}, n^{i 1}\right)_{L^{2}(\Omega)}+\left(\eta^{i 2}, n^{i 2}\right)_{L^{2}(\Omega)}\right)+\mathbf{i}\left(\left(\eta^{i 2}, n^{i 1}\right)_{L^{2}(\Omega)}-\left(\eta^{i 1}, n^{i 2}\right)_{L^{2}(\Omega)}\right) .
$$

For the space $\eta^{i}=\eta^{i 1}+\mathbf{i} \eta^{i 2} \in \mathbb{H}_{0}^{1}(\Omega)$, define its norm as

$$
\left\|\eta^{i}\right\|_{\mathbb{H}_{0}^{1}(\Omega)}=\left(\left\|\eta^{i 1}\right\|_{H_{0}^{1}(\Omega)}^{2}+\left\|\eta^{i 2}\right\|_{H_{0}^{1}(\Omega)}^{2}\right)^{\frac{1}{2}}
$$

If $\nabla \eta^{i}=\nabla \eta^{i 1}+i \nabla \eta^{i 2}$, then the definition (3) is equivalent to $\left\|\eta^{i}\right\|_{\mathbb{H}_{0}^{1}(\Omega)}=\left(\left\|\nabla \eta^{i 1}\right\|_{\mathbb{L}^{2}(\Omega)}^{2}+\right.$ $\left.\left\|\nabla \eta^{i 2}\right\|_{\mathbb{L}^{2}(\Omega)}^{2}\right)^{\frac{1}{2}}$. If $\eta^{i}, n^{i} \in \mathbb{H}_{0}^{1}(\Omega)$, then the inner product of space $\mathbb{H}_{0}^{1}(\Omega)$ is defined as

$$
\left(\eta^{i}, n^{i}\right)_{\mathbb{H}_{0}^{1}(\Omega)}=\left(\eta^{i 1}, n^{i 1}\right)_{H_{0}^{1}(\Omega)}+\left(\eta^{i 2}, n^{i 2}\right)_{H_{0}^{1}(\Omega)} .
$$

The definition is equivalent to $\left(\eta^{i}, n^{i}\right)_{\mathbb{H}_{0}^{1}(\Omega)}=\left(\nabla \eta^{i 1}, \nabla n^{i 1}\right)_{\mathbb{L}^{2}(\Omega)}+\left(\nabla \eta^{i 2}, \nabla n^{i 2}\right)_{\mathbb{L}^{2}(\Omega)}$.

Definition. The Hilbert space $W(0, T)$ is called solution space, defined by

$$
W(0, T)=\left\{\eta^{i} \mid \eta^{i} \in L^{2}(0, T ; \mathbb{V}), \eta^{i \prime} \in L^{2}\left(0, T ; \mathbb{V}^{\prime}\right)\right\}
$$

Then the norm of $W(0, T)$ can be defined as

$$
\left\|\eta^{i}\right\|_{W(0, T)}=\left(\left\|\eta^{i}\right\|_{L^{2}(0, T ; \mathbb{V})}^{2}+\left\|\eta^{i \prime}\right\|_{L^{2}\left(0, T ; \mathbb{V}^{\prime}\right)}^{2}\right)^{\frac{1}{2}}
$$


If $\eta^{i}, n^{i} \in W(0, T)$, then the definition of its inner product is given by

$$
\left(\eta^{i}, n^{i}\right)_{W(0, T)}=\left(\eta^{i}, n^{i}\right)_{\mathbb{V}}+\left(\eta^{i \prime}, n^{i \prime}\right)_{\mathbb{V}^{\prime}}
$$

Denotes the continuous functions set $C(0, T ; \mathbb{H})$, and spaces $L^{2}(0, T ; \mathbb{H}), L^{2}(0, T ; \mathbb{V}), L^{2}\left(0, T ; \mathbb{V}^{\prime}\right)$. Further, $\mathbb{V}^{\prime}$ is the complex conjugate space of $\mathbb{V}$. Certainly, $(\mathbb{V}, \mathbb{H})$ is also a Gelfand triple spaces $\mathbb{V} \hookrightarrow \mathbb{H} \hookrightarrow \mathbb{V}^{\prime}$, in which two embeddings are continuous, dense and compact.

\section{Solution of nonlinear Schrödinger equation}

Definition 2. A function $\eta^{i}$ is said as weak solution of system in the form of time-depended Schrödinger equation (1) for $i$-th particle $P^{i}, i=1,2, \cdots, N$, if $\eta^{i} \in W(0, T)$ and satisfy

$$
\begin{aligned}
& \int_{0}^{T} \int \mathbf{i} \frac{\partial \eta^{i}(t, \mathbf{r})}{\partial t} \sigma^{i} d \mathbf{r} d t \\
= & -\frac{1}{2} \int_{0}^{T} \int \nabla \eta^{i}(t, \mathbf{r}) \nabla \sigma^{i} d \mathbf{r} d t+\int_{0}^{T} \int[e(t, \mathbf{r})+V(t, \mathbf{r})-g(t) \mathbf{r}] \eta^{i}(t, \mathbf{r}) \sigma^{i} d \mathbf{r} d t,
\end{aligned}
$$

where $\forall \sigma^{i} \in \mathcal{D}^{\prime}(0, T)$ by the means of distribution space, $\sigma^{i} \in C^{1}(0, T ; \mathbb{V})$ and $\sigma^{i}(T)=0$.

Theorem 3. For $\eta^{i}(0, \mathbf{r})=\eta_{0}^{i}(\mathbf{r}) \in \mathbb{V}$ of particle $P^{i}, i=1,2, \cdots, N$, then there exists a unique solution $\eta^{i}(t, \mathbf{r}) \in W(0, T)$ for time-depended Schrödinger system (1).

Proof. Faedo-Galerkin method is using to construct an approximate solution for system (1). From Gelfand triple spaces, $\mathbb{V} \hookrightarrow \mathbb{H}$ is compact, then there exists an orthogonal basis of $\mathbb{H},\left\{w^{j}\right\}_{j=1}^{\infty}$ consisting of eigenfunctions of $A=-\Delta$, such that $A w^{j}=\lambda^{j} w^{j}$ for all $j$, $0<\lambda^{1} \leq \lambda^{2} \leq, \cdots, \lambda^{j} \rightarrow \infty$ as $j \rightarrow \infty$. $G^{i}$ is the orthogonal projection of $\mathbb{H}$ (or $\mathbb{V}$ ) onto the space spanned by $\left\{w^{1}, \cdots, w^{i^{\prime}}\right\}$. For $1 \leq i \leq N$, an approximate solution is defined for equation (1) by $\tilde{\eta}^{i j^{\prime}}(t)=\sum_{j=1}^{j^{\prime}} a^{i j}(t) w^{j}$, and $a^{i j}(t)$ is real-valued coefficient function. From $\mathbf{n}(t, \mathbf{r})=\sum_{i=1}^{N}\left|\eta^{i}(t, \mathbf{r})\right|^{2}$ to get expansion of $\tilde{e}(t, \mathbf{r})$ and $\tilde{V}(t, \mathbf{r})$ of $e(t, \mathbf{r})$ and $V(t, \mathbf{r})$ using $\left\{w^{j}\right\}$. Taking $\sigma^{i}=w^{j}$ at weak form (4), then approximate solution $\tilde{\eta}^{i j^{\prime}}(t)$ satisfy ordinary 
differential equation as

$\left\{\begin{array}{l}\int_{0}^{T} \int \mathbf{i} \frac{\partial \tilde{\eta}^{i j^{\prime}}}{\partial t} w^{j} d \mathbf{r} d t=-\frac{1}{2} \int_{0}^{T} \int \nabla \tilde{\eta}^{i j^{\prime}} \nabla w^{j} d \mathbf{r} d t+\int_{0}^{T} \int[\tilde{e}(t, \mathbf{r})+\tilde{V}(t, \mathbf{r})-g(t) \mathbf{r}] \tilde{\eta}^{i j^{\prime}} w^{j} d \mathbf{r} d t \\ \tilde{\eta}^{i j^{\prime}}(0)=\tilde{\eta}_{0}^{i}, \quad 1 \leq i \leq N .\end{array}\right.$

Substitute $\tilde{\eta}^{i j^{\prime}}$ as approximate form in (5), from standard theory of ODE, ensure a unique local solution $\left\{\tilde{\eta}^{i j^{\prime}}\right\}, i=1,2, \cdots, N$ for approximate system (5). For $\tilde{\eta}_{0}^{i j^{\prime}}$, there exists a $\bar{\eta}_{0}^{i} \in \mathbb{V}$ such that

$$
\tilde{\eta}_{0}^{i j^{\prime}} \rightarrow \bar{\eta}_{0}^{i} \text { strongly in } \quad \mathbb{H}_{0}^{1}(\Omega), \quad \text { as } j^{\prime} \rightarrow \infty
$$

Take analogous argument for $\left\{\tilde{\eta}^{i j^{\prime}}\right\}$, there exist a function $\bar{\eta}^{i} \in \mathbb{V}$, that $\left\|\tilde{\eta}^{i j^{\prime}}-\bar{\eta}^{i}\right\|_{\mathbb{V}} \rightarrow 0$ as $j^{\prime} \rightarrow \infty$. That is, approximate solution $\tilde{\eta}^{i j^{\prime}}$ is bounded in $L^{\infty}(0, T ; \mathbb{V})$. Suppose that $\left\{\tilde{\eta}^{i j^{\prime}}\right\}$ and $\left\{\tilde{\eta}^{j j^{\prime}}\right\}$ are two solutions of equation (1) for initial guess $\eta_{0}^{i}$ and $\eta_{0}^{j}, \tilde{e}^{i j^{\prime}}=\tilde{e}\left(t, \mathbf{r}, \eta^{i j^{\prime}}\right), \tilde{V}^{i j^{\prime}}=$ $\tilde{V}\left(t, \mathbf{r}, \eta^{i j^{\prime}}\right)$ for solution $\eta^{i j^{\prime}}, \tilde{e}^{j j^{\prime}}=\tilde{e}\left(t, \mathbf{r}, \eta^{j j^{\prime}}\right), \tilde{V}^{j j^{\prime}}=\tilde{V}\left(t, \mathbf{r}, \eta^{j j^{\prime}}\right)$ for solution $\eta^{j j^{\prime}}$. Then to have

$$
\left\|\tilde{e}^{i j^{\prime}} \tilde{\eta}^{i j^{\prime}}-\tilde{e}^{j j^{\prime}} \tilde{\eta}^{j j^{\prime}}\right\|^{2} \leq\left\|\tilde{e}^{i j^{\prime}}\right\|^{2}+\left\|\tilde{\eta}^{i j^{\prime}}-\tilde{\eta}^{j j^{\prime}}\right\|^{2}+\left\|\tilde{e}^{i j^{\prime}}-\tilde{e}^{j j^{\prime}}\right\|^{2}+\left\|\tilde{\eta}^{j j^{\prime}}\right\|^{2}
$$

There exist $C^{\prime}(t)$ such that

$$
\left\|\tilde{e}^{i j^{\prime}}-\tilde{e}^{j j^{\prime}}\right\|_{\mathbb{H}}^{2} \leq \int \frac{\sum_{i=1}^{N}\left\|\tilde{\eta}^{i j^{\prime}}+\tilde{\eta}^{j j^{\prime}}\right\|_{\mathbb{H}}^{2}\left\|\eta^{i j^{\prime}}-\eta^{j j^{\prime}}\right\|_{\mathbb{H}}^{2}}{\left|\mathbf{r}-\mathbf{r}^{\prime}\right|} d \mathbf{r}^{\prime} \leq C^{\prime}(t)\left\|\tilde{\eta}^{i j^{\prime}}-\tilde{\eta}^{j j^{\prime}}\right\|_{\mathbb{H}}^{2}
$$

as $j^{\prime} \rightarrow \infty$. Further,

$$
\left\|\tilde{V}^{i j^{\prime}} \tilde{\eta}^{i j^{\prime}}-\tilde{V}^{j j^{\prime}} \tilde{\eta}^{j j^{\prime}}\right\|^{2} \leq\left\|\tilde{V}^{i j^{\prime}}\right\|_{\mathbb{H}}^{2}+\left\|\tilde{\eta}^{i j^{\prime}}-\tilde{\eta}^{j j^{\prime}}\right\|_{\mathbb{H}}^{2}+\left\|\tilde{V}^{i j^{\prime}}-\tilde{V}^{j j^{\prime}}\right\|_{\mathbb{H}}^{2}+\left\|\tilde{\eta}^{j j^{\prime}}\right\|_{\mathbb{H}}^{2}
$$


There exist $C^{\prime \prime}(t)$ such that

$$
\left.\left\|\tilde{V}^{i j^{\prime}}-\tilde{V}^{j j^{\prime}}\right\|_{\mathbb{H}}^{2} \leq \frac{5}{3} c_{k} \int\left(\sum_{i=1}^{N}\left|\tilde{\eta}^{i j^{\prime}}(t, \mathbf{r})\right|^{2}\right)^{2 / 3}-\left(\sum_{j=1}^{N}\left|\tilde{\eta}^{j j^{\prime}}(t, \mathbf{r})\right|^{2}\right)^{2 / 3}\right) d \mathbf{r} \leq C^{\prime \prime}(t)\left\|\tilde{\eta}^{i j^{\prime}}-\tilde{\eta}^{j j^{\prime}}\right\|_{\mathbb{H}}^{2} .
$$

Denote $\|\mathbf{r}\|$ as norm of vector $\mathbf{r}$, hence, from weak form (4) to get

$$
\begin{aligned}
& \frac{1}{2} \frac{d}{d t}\left\|\tilde{\eta}^{i j^{\prime}}-\tilde{\eta}^{j j^{\prime}}\right\|_{\mathbb{H}}^{2}+g(t)\|\mathbf{r}\|\left\|\tilde{\eta}^{i j^{\prime}}-\tilde{\eta}^{j j^{\prime}}\right\|_{\mathbb{V}}^{2} \\
\leq & -\frac{1}{2}\left\|\tilde{\eta}^{i j^{\prime}}-\tilde{\eta}^{j j^{\prime}}\right\|_{\mathbb{V}}^{2}+\left\|\tilde{e}^{i j^{\prime}}\right\|_{\mathbb{H}}^{2}+\left\|\tilde{\eta}^{i j^{\prime}}-\tilde{\eta}^{j j^{\prime}}\right\|_{\mathbb{H}}^{2}+\left\|\tilde{e}^{i j^{\prime}}-\tilde{e}^{j j^{\prime}}\right\|_{\mathbb{H}}^{2}+\left\|\tilde{\eta}^{j j^{\prime}}\right\|_{\mathbb{H}}^{2} \\
& +\left\|\tilde{V}^{i j^{\prime}}\right\|_{\mathbb{H}}^{2}+\left\|\tilde{\eta}^{i j^{\prime}}-\tilde{\eta}^{j j^{\prime}}\right\|_{\mathbb{H}}^{2}+\left\|\tilde{V}^{i j^{\prime}}-\tilde{V}^{j j^{\prime}}\right\|_{\mathbb{H}}^{2}+\left\|\tilde{\eta}^{j j^{\prime}}\right\|_{\mathbb{H}}^{2} \cdot \\
\leq & -\frac{1}{2}\left\|\tilde{\eta}^{i j^{\prime}}-\tilde{\eta}^{j j^{\prime}}\right\|_{\mathbb{V}}^{2}+\left(1+C^{\prime}(t)+C^{\prime \prime}(t)\right)\left\|\tilde{\eta}^{i j^{\prime}}-\tilde{\eta}^{j j^{\prime}}\right\|_{\mathbb{H}}^{2} \\
& +\left(\left\|\tilde{e}^{i j^{\prime}}\right\|_{\mathbb{H}}^{2}+\left\|\tilde{V}^{i j^{\prime}}\right\|_{\mathbb{H}}^{2}+\left\|\tilde{\eta}^{j j^{\prime}}\right\|_{\mathbb{H}}^{2}\right)
\end{aligned}
$$

Set $I^{i j}(t)=\left(1+C^{\prime}(t)+C^{\prime \prime}(t)\right)\left\|\tilde{\eta}^{i j^{\prime}}-\tilde{\eta}^{j j^{\prime}}\right\|_{\mathbb{H}}^{2},\left(g(t)\|\mathbf{r}\|+\frac{1}{2}\right)\left\|\tilde{\eta}^{i j^{\prime}}-\tilde{\eta}^{j j^{\prime}}\right\|_{\mathbb{V}}^{2} \geq 0$, and $C(t)=$ $\left\|\tilde{e}^{i j^{\prime}}\right\|_{\mathbb{H}}^{2}+\left\|\tilde{V}^{i j^{\prime}}\right\|_{\mathbb{H}}^{2}+\left\|\tilde{\eta}^{j j^{\prime}}\right\|_{\mathbb{H}}^{2}$ to get

$$
\frac{d}{d t} I^{i j}(t) \leq I^{i j}(t)+C(t)
$$

Set $I^{i j}(0)=\left(1+C^{\prime}(0)+C^{\prime \prime}(0)\right)\left\|\tilde{\eta}_{0}^{i j^{\prime}}-\tilde{\eta}_{0}^{j j^{\prime}}\right\|_{\mathbb{H}}^{2}$, Gronwall inequality yield that

$$
I^{i j}(t) \leq e^{t} I^{i j}(0)+\int_{0}^{t} C(t) e^{t-t^{\prime}} d t^{\prime}, \quad 0 \leq t^{\prime} \leq t .
$$

Therefore, $\tilde{\eta}_{0}^{i j^{\prime}} \rightarrow \tilde{\eta}_{0}^{j j^{\prime}}$ implied $\tilde{\eta}^{i j^{\prime}} \rightarrow \tilde{\eta}^{j j^{\prime}}$ in $L^{2}(0, T ; \mathbb{H})$ as $j^{\prime} \rightarrow \infty$, and same argument to $L^{2}(0, T ; \mathbb{V})$ as $j^{\prime} \rightarrow \infty$ from (6). Taking $\tilde{\eta}^{j j^{\prime}}=\eta^{i}$ to find that $\tilde{\eta}^{i j^{\prime}} \rightarrow \eta^{i}$ in $C(0, T ; \mathbb{H})$. Thus, the inclusion $C(0, T ; \mathbb{H}) \subset W(0, T)$ to ensure Theorem 1. 


\section{Control theory for particle at surface}

Consider control function $g(t)$, and its quantum optimal control $g^{*}(t)$. Suppose $\mathcal{G}=L^{2}(0, T)$ is the space of control $g(t)$, and $\mathcal{G}_{a d}$ is a admissible set of $\mathcal{G}$. Set ground state of $i$-th particle $P^{i}$ as $\eta^{i}(0)=\eta_{0}$. Consider cost function of $i$-th particle $P^{i}$ for time-depended Schrödinger system (1) is in the form of

$$
J^{i}(g)=\epsilon^{1}\left\|\eta_{f}^{i}(g)-\eta_{d}^{i}\right\|_{\mathbb{H}}^{2}+\epsilon^{2}\|g\|_{\mathcal{G}}^{2}
$$

In criteria function (7), $\forall g \in \mathcal{G}_{a d}, \eta_{d}^{i}$ is target state, $\left.\eta_{f}^{i}(g)\right)$ is observed final state of particle $P^{i}$ at final time $t_{f} \cdot \epsilon^{i}, i=1,2$ are weighted coefficients for balancing the values of inherent cost and running cost. Total cost function $J(g)=\sum_{i=1}^{N} J^{i}(g)$ for all particles at a surface.

Two fundamental problems of quantum optimal control for system (1):

i). find quantum optimal control $g^{*}$ for system (1).

ii). find Eular-Lagrange system for $g^{*}$.

For $i$-th particle $P^{i}, g^{*}(t)$ is quantum optimal control for system (1) subject to cost function $(7)$.

Theorem 4. For $\eta_{0}^{i} \in \mathbb{V}$ of $i$-th particle $P^{i}, i=1,2, \cdots, N$, if $\mathcal{G}_{a d}$ is closed convex (bounded) admissible subset of $\mathcal{G}$, then there exists at least one quantum optimal control $g^{*}$ of timedepended Schrödinger system (1) subject to cost function (7).

Proof. Set $J=\inf _{g \in \mathcal{G}_{a d}} J^{i}(g)$, since $\mathcal{G}_{a d}$ is non-empty, there is a sequence $\left\{g^{k}\right\}$ in $\mathcal{G}_{a d}$ such that $\inf _{g \in \mathcal{G}_{a d}} J^{i}(g)=\lim _{k \rightarrow \infty} J\left(g^{k}\right)=J$. Since $\left\{J^{i}(g)\right\}$ is bounded in $\mathbf{R}^{+}$, and $\mathcal{G}_{a d}$ is closed and convex (bounded) subset of $\mathcal{G}$, there exist a subsequences $\left\{g^{k^{\prime}}\right\}$ of $\left\{g^{k}\right\}$ can be extracted, and exist a $g^{*} \in \mathcal{G}_{a d}$, such that

$$
g^{k^{\prime}} \rightarrow g^{*} \text { weakly in } \mathcal{G} \quad \text { as } k^{\prime} \rightarrow \infty
$$


From existence theorem of weak solution to get estimate $\left\|\eta^{i}\right\|_{\mathbb{H}}^{2}+\left\|\eta^{i}\right\|_{\mathbb{V}}^{2}$ is bounded for $\eta^{i}$ of $i$-th particle $P^{i}$. For control $g^{k^{\prime}}$, from boundedness of $\mathcal{G}_{a d}$ that

$$
\eta^{i}\left(g^{k^{\prime}}\right) \text { is bounded in } L^{2}(0, T ; \mathbb{H}) \cap L^{2}(0, T ; \mathbb{V}) \text {. }
$$

Setting $\eta^{i *}=\eta^{i}\left(g^{*}\right)$, there exist a subsequence $\left\{\eta^{i}\left(g^{k^{\prime \prime}}\right)\right\}$ of $\left\{\eta^{i}\left(g^{k^{\prime}}\right)\right\}$, and a $\eta^{i *} \in W(0, T)$ such that

$$
\eta^{i}\left(g^{k^{\prime \prime}}\right) \rightarrow \eta^{i *} \quad \text { weakly in } L^{2}(0, T ; \mathbb{H}) \cap L^{2}(0, T ; \mathbb{V}) .
$$

as $k^{\prime \prime} \rightarrow \infty$. Since the embedding $\mathbb{V} \hookrightarrow \mathbb{H}$ is compact, from Aubin-Lions-Temam theorem, then there is $\bar{\eta}^{i} \in \mathbb{H}$ that

$$
\eta^{i}\left(g^{k^{\prime \prime}}\right) \rightarrow \bar{\eta}^{i} \quad \text { strongly in } L^{2}(0, T ; \mathbb{H}), \quad \text { as } k^{\prime \prime} \rightarrow \infty,
$$

and get the convergences for $\eta^{i}\left(g^{k^{\prime \prime}}\right)$ as

$$
\begin{cases}\frac{\partial \eta^{i}\left(g^{k^{\prime \prime}}\right)}{\partial t} \rightarrow \frac{\partial \bar{\eta}^{i}}{\partial t} & \text { weakly in } L^{2}\left(0, T ; \mathbb{V}^{\prime}\right) \\ \nabla \eta^{i}\left(g^{k^{\prime \prime}}\right) \rightarrow \nabla \bar{\eta}^{i} & \text { weakly in } L^{2}(0, T ; \mathbb{H})\end{cases}
$$

as $k^{\prime \prime} \rightarrow \infty$. Set $\eta^{i k^{\prime \prime}}=\eta^{i}\left(g^{k^{\prime \prime}}\right), \eta_{0}^{i k^{\prime \prime}}=\eta_{0}^{i}$, therefore, for $i$-th particle $P^{i}$, by taking test function $w^{j}=\sigma^{i}$ in (5), by the definition of weak form (4) to find

$$
\left\{\begin{array}{l}
\int_{0}^{T} \int \mathbf{i} \frac{\partial \eta^{i k^{\prime \prime}}}{\partial t} \sigma^{i} d \mathbf{r} d t=-\frac{1}{2} \int_{0}^{T} \int \nabla \eta^{i k^{\prime \prime}} \nabla \sigma^{i} d \mathbf{r} d t+\int_{0}^{T} \int[e(t, \mathbf{r})+V(t, \mathbf{r})-g(t) \mathbf{r}] \eta^{i k^{\prime \prime}} \sigma^{i} d \mathbf{r} d t \\
\eta^{i k^{\prime \prime}}(0)=\eta_{0}^{i k^{\prime \prime}}, \quad 1 \leq i \leq N .
\end{array}\right.
$$

If using (8), (9), and taking $k^{\prime \prime} \rightarrow \infty$ to yield that

$$
\left\{\begin{array}{l}
\int_{0}^{T} \int \mathbf{i} \frac{\partial \bar{\eta}^{i}}{\partial t} \sigma^{i} d \mathbf{r} d t=-\frac{1}{2} \int_{0}^{T} \int \nabla \bar{\eta}^{i} \nabla \sigma^{i} d \mathbf{r} d t+\int_{0}^{T} \int[e(t, \mathbf{r})+V(t, \mathbf{r})-g(t) \mathbf{r}] \bar{\eta}^{i} \sigma^{i} d \mathbf{r} d t \\
\bar{\eta}^{i}(0)=\eta_{0}^{i}, \quad 1 \leq i \leq N, \quad \forall \sigma^{i} \in C^{1}(0, T ; \mathbb{V}) .
\end{array}\right.
$$


It inferred that $\bar{\eta}^{i}$ is a solution of (1) in the sense of distribution $\mathcal{D}^{\prime}(0, T)$ on $(0, T)$. From uniqueness of weak solution for system (1) to attain $\bar{\eta}^{i}=\eta^{i}\left(g^{*}\right)$. That is, for $\eta^{i}$ to get two convergences as

$$
\begin{aligned}
& \eta^{i}\left(g^{k^{\prime \prime}}\right) \rightarrow \eta^{i}\left(g^{*}\right) \quad \text { strongly in } \quad L^{2}(0, T ; \mathbb{H}), \\
& \eta_{f}^{i}\left(g^{k^{\prime \prime}}\right) \rightarrow \eta_{f}^{i}\left(g^{*}\right) \quad \text { strongly in } \quad \mathbb{H},
\end{aligned}
$$

as $k^{\prime \prime} \rightarrow \infty$. Since the norm $\|\cdot\|_{\mathbb{L}^{2}(\Omega)}$ are lower semi-continuous in weak topology of $\mathbb{L}^{2}(\Omega)$, for $i$-th particle $P^{i} \lim _{k^{\prime \prime} \rightarrow \infty} \inf \left\|\eta_{f}^{i}\left(g^{k^{\prime \prime}}\right)-\eta_{d}^{i}\right\|_{\mathbb{H}}^{2} \geq\left\|\eta_{f}^{i}\left(g^{*}\right)-\eta_{d}^{i}\right\|_{\mathbb{H}}^{2}$. Vice versa, from weak convergence (8) that $\liminf _{k^{\prime \prime} \rightarrow \infty}\left(g^{k^{\prime \prime}}, g^{k^{\prime \prime}}\right)_{\mathcal{G}} \geq\left(g^{*}, g^{*}\right)_{\mathcal{G}}$. For cost function $(7), J=\liminf _{k^{\prime \prime} \rightarrow \infty} J^{i}\left(g^{k^{\prime \prime}}\right) \geq$ $J^{i}\left(g^{*}\right)$, and $J^{i}\left(g^{*}\right)=\inf _{g \in \mathcal{G}_{a d}} J^{i}(g)$. That is, $g^{*}$ is quantum optimal control of time-depended Schrödinger system (1) subject to criteria function (7). It is Theorem 4.

Theorem 5. For $\eta_{0}^{i} \in \mathbb{V}$ of control problem for system (1) to cost function (7), if $\mathcal{G}_{a d}$ is closed convex (bounded) admissible subset of $\mathcal{G}$, then quantum optimal control $g^{*}$ is characterized by Eular-Lagrange system as

$$
\begin{aligned}
& \left\{\begin{array}{l}
\mathbf{i} \frac{\partial}{\partial t} \eta^{i}(t, \mathbf{r})=-\frac{1}{2} \nabla^{2} \eta^{i}(t, \mathbf{r})+\left[e(t, \mathbf{r})+V(t, \mathbf{r})-g^{*}(t) \mathbf{r}\right] \eta^{i}(t, \mathbf{r}) \quad \text { in } \mathrm{Q}, \\
\eta^{i}\left(0, g^{*}\right)=\eta_{0}^{i} \quad \text { on } \Omega .
\end{array}\right. \\
& \left\{\begin{array}{c}
\mathbf{i} \frac{\partial}{\partial t} n^{i}(t, \mathbf{r})=-\frac{1}{2} \nabla^{2} n^{i}(t, \mathbf{r})+[e(t, \mathbf{r})+V(t, \mathbf{r})] n^{i}(t, \mathbf{r}) \quad \text { in } \mathrm{Q}, \\
\mathbf{i} n^{i}\left(T, g^{*}\right)=\eta_{f}^{i}\left(g^{*}\right)-\eta_{d}^{i} \quad \text { on } \Omega .
\end{array}\right. \\
& \int_{0}^{T} \int \sigma^{*} n^{i}\left(g^{*}\right)\left(g-g^{*}\right) d \mathbf{r} d t+\left(g^{*}, g-g^{*}\right)_{\mathcal{G}} \geq 0 \quad \forall g \in \mathcal{G}_{a d} .
\end{aligned}
$$

In here, $n^{i}(t, \mathbf{r}) \in W(0, T)$ is solution of adjoint system corresponding to $\eta^{i}(t, \mathbf{r})$ of timedepended Schrödinger system (1). (10) is necessary optimality condition for quantum optimal control $g^{*}$ of $i$-th particle $P^{i}, i=1,2, \cdots, N$. $\sigma^{*}$ is conjugate operator of $\sigma$ in $\sigma\left(\eta^{i}\right)=g^{*} \eta^{i}$. 


\section{Numerical approach for one particle at matter surface}

For $N=1$ one particle case, the interactions of particles are disappeared, and first term remain at the $V(t, \mathbf{r})$ of TF model in (2). Domain $\Omega=[0, R], Q=[0, T] \times \Omega,(t, \mathbf{r}) \in Q$ and $T=300, R=100$. Reduced Planck constant $\hbar=1.0545715964207855 \times 10^{34}$ js and electron mass $m=9.10938188 \times 10^{-31} \mathrm{~kg}$. Start control function is set as $g_{0}(t)=1.0 \times 10^{-9} \sin (500 t)$. The polar plot of $g_{0}(t)$ is in the Figure 2 .

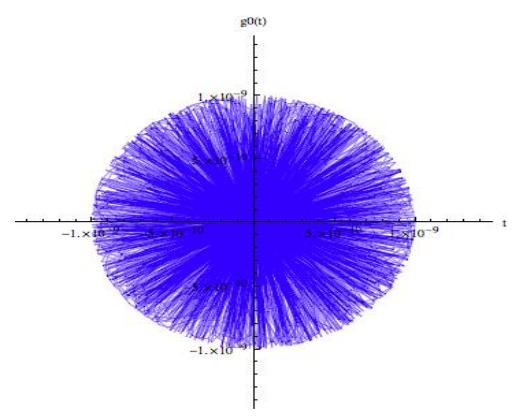

Figure 2: Polar plot of start control $g_{0}(t)$

After the iterations, the stop step $n^{*}=9$, the obtained optimal control function $g^{*}(t)=$ $9.2464 \times 10^{-11}+1.0 \times 10^{-9} \sin (500 t)$. The polar plot are in the Figure 3. The density

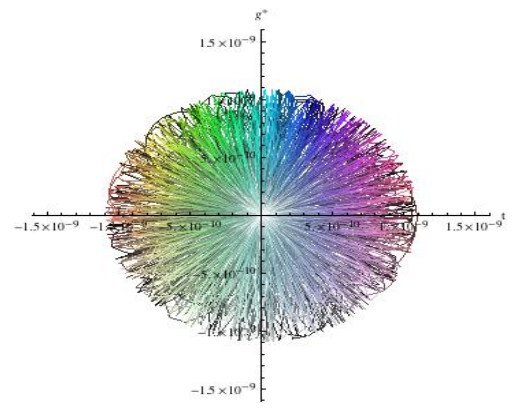

Figure 3: Polar plot of optimal control $g^{*}(t)$

function plot at $[0,300] \times[0,100]$ are in the Figure 4 and Figure 5. Optimal cost function values $J\left(g^{*}\right)=1.26135 \times 10^{7}$. The cost function values are each iteration: $6.05348 \times 10^{7}$, $1.09076 \times 10^{7}, 7.1184 \times 10^{6}, 1.51325 \times 10^{7}, 1.35285 \times 10^{7}, 2.72698 \times 10^{7}, 1.57147 \times 10^{7}, 5.9729$ $\times 10^{7}, 1.26135 \times 10^{7}$. Plot in Figure 6. 


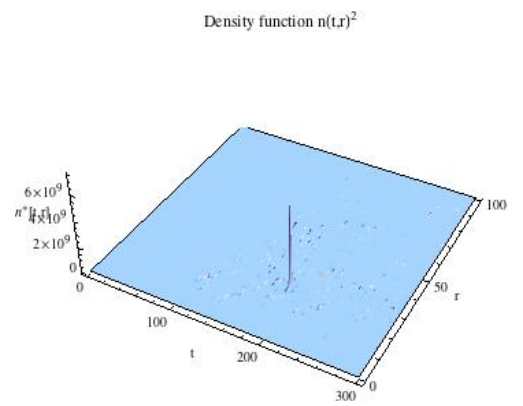

Figure 4: Plot3D of optimal density function $\mathbf{n}^{*}(t, r)^{2}$

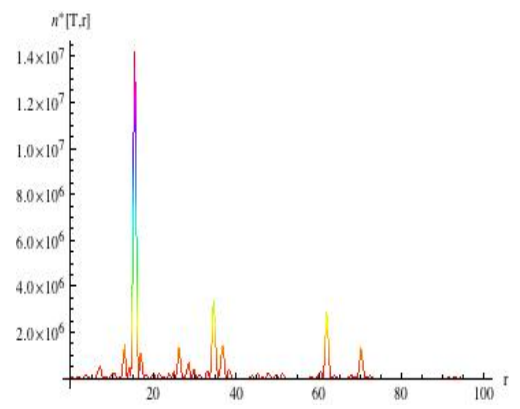

Figure 5: Plot of optimal density function $\mathbf{n}^{*}(T, r), T=300$

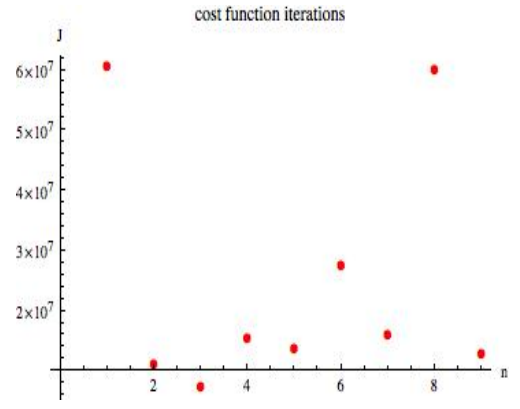

Figure 6: Plot of cost unction $J\left(g^{*}(t)\right)$

The error of cost function values $e J=J(g)_{n}-J(g)_{n-1}, n=1,2, \cdots, 9$ are each iteration: $6.05348 \times 10^{7},-4.96272 \times 10^{7},-3.78924 \times 10^{6}, 8.0141 \times 10^{6},-1.60403 \times 10^{6}, 1.37414 \times 10^{7}$, $-1.15551 \times 10^{7}, 4.40143 \times 10^{7},-4.71155 \times 10^{7}$.

CPU running time is 234.797 seconds, the total used memory is 1470166272 bytes. The results of Figure 5 and Figure 6 in numerical approach are accordance to the current litera- 
tures of physics field.

\section{Conclusions and discussions}

In this work, we investigate theoretical and computational quantum control for particles at matter surface using density function theory. For time- depended Schrödinger equation of particles, control theory is applied to consider the quantum control of electron motion surrounding particles. For one particle case in 3D, numerical control is executed for TF model at real physical scale. In comprising to existing results of physical experiments to conclude that: i). optimal control theory is worked to manipulate the particles appeared at matter surface. ii). density function theory (DFT) as a fresh tool can be using to do control at scale of molecule, atom and nuclei. Future work is for computational experiment control of multi-particles, poly-atoms, and large number of particles at surface.

\section{Acknowledgement}

The author sincere thanks to 249th ACS National Meeting \& Exposition 2015 for poster 370 at PHYS Poster session.

\section{References}

(1) Q. F. Wang, Quantum control at matter surface, ACS National Meeting \& Exposition 2009, Poster.

(2) Q. F. Wang, Quantum control for particles at matter surface, ACS National Meeting \& Exposition 2014, Poster.

(3) Q. F. Wang, Quantum numerical control for particles at matter surface, 249th ACS National Meeting \& Exposition 2015, Poster. 
(4) S. Horikoshi, N. Serpone, Introduction to Nano Particles, Microwaves in Nano-particles, Wiley-VCH Verlag GmbH \& Co KGaA, 2013.

(5) H. G Mack, Combined quantum mechanics and classical electrodynamics mulit-scale approach for the calculation of SERS spectra (A brief survey), 2013.

(6) W. Kohn, I, J. Sham, Self-consistent equations including exchange and correlation effects, Physical Review 140, A1133, 1965.

(7) P. Hohenberg, W. Kohn, Inhomogeneous electron gad, Physical Review 136, B864, 1964.

(8) L. H. Thomas, The calculation of atomic fields, Proc. Camb. Phil. Soc. 23, 542, 1927.

(9) P. A. M. Dirac, Note on exchange phenomena in the Thomas atom, Proc. Camb. Phil. Soc. 26, 376, 1930 .

(10) E. Fermi, Un Metodo Statistico per la Determinazione di alcune Prioprietà dell'Atomo, Rend. Accad. Naz. Lincei 6,602, 1927.

(11) C. F. Weizsäcker, Zur Theorie der Kernmassen, Zeitschrift für Physik 96 (7-8) 431, 1935.

(12) N. M. Harrison, An Introduction to Density Function Theory, 2002.

(13) J. D. Morgan III, Thomas-Fermi and other density function theories, Atomic, Molecular, \& Optical Physics Handbooks, 1996. 